\title{
Estudo da fotoeletrodeposição de partículas de cobre sobre filmes de diamante dopado com boro
}

\author{
Photoelectrodeposition study of copper particles on boron doped diamond films
}

Mildred C.E .Ribeiro' ${ }^{1}$, Andrea B. Couto ${ }^{1}$, Fernanda L. Migliorini ${ }^{1}$, Neidenei G.Ferreira ${ }^{1}$, Maurício R. Baldan ${ }^{1}$

\begin{abstract}
Resumo
A fotoeletrodeposição de partículas de cobre sobre filmes de diamante dopado com boro (DDB) com diferentes níveis de dopagem foi investigada. Ênfases foram dadas no estudo da influência do nível de dopagem dos filmes e na eletrodeposição fotoassistida de cobre utilizando soluções com diferentes pHs (ácido e neutro). Os filmes crescidos foram caracterizados por espectroscopia Raman e a concentração de portadores foi estimada por medidas de Mott-Schottky Plot. Os resultados mostraram que a deposição de partículas de $\mathrm{Cu}$ sobre os filmes de DDB apresentou maior homogeneidade nos filmes com maior dopagem $(\mathrm{B} / \mathrm{C}=30000 \mathrm{ppm})$ quando comparados aos filmes de menor dopagem $(\mathrm{B} / \mathrm{C}=5000 \mathrm{ppm})$. Essa diferença na homogeneidade pode ser atribuída à melhor condutividade do eletrodo mais dopado devido à maior quantidade de boro presente neste filme, potencializando o processo de redução do cobre. Com relação ao meio eletrolítico (ácido, neutro) utilizado nas deposições fotoassistidas, foi observado, em meio neutro, uma melhor eficiência na deposição de $\mathrm{Cu}$. Este comportamento pode estar associado à menor competição entre o $\mathrm{H}^{+}$e o $\mathrm{Cu}^{2+}$ pelos elétrons fotogerados, uma vez que o $\mathrm{H}^{+}$pode ser fotoeletricamente reduzido na superfície do diamante.
\end{abstract}

Palavras-chave: BDD; Fotoeletrodeposição; Cobre.

\begin{abstract}
Copper photoelectrodeposition on boron-doped diamond (BDD) films with different doping levels was investigated. Emphases were given to study the influence of the doping level of the films and of the $\mathrm{Cu}$ photoassisted electrodeposition processes using solutions with different pHs (acid and neutral). The films were characterized by Raman spectroscopy and the acceptor densities were estimated by Mott-Schottky Plot. The results showed that the Cu deposits presented more homogeneity for highly doped $B D D$ electrode $(B / C=30000 \mathrm{ppm})$ when compared to the slightly doped $B D D$ electrode $(B / C=5000 \mathrm{ppm})$. This difference in the uniformity can be attributed to the boron amount present in this electrode, where the conductivity is better and, consequently, improves the Cu reduction process. Concerning to the different electrolytes used in this work, a better efficiency of Cu photoelectrodeposition process was obtained by using neutral solution. This behavior may be attributed to the lower competition between $\mathrm{H}^{+}$and $\mathrm{Cu}^{2+}$ by the photogenerated electron, since $\mathrm{H}^{+}$ can be photoelectroreduced on the surface of the diamond.
\end{abstract}

Keywords: EDD; Photoelectrodeposition; Copper.

${ }^{1}$ Instituto Nacional de Pesquisas Espaciais - São José dos Campos/SP - Brasil.

Autor correspondente: Andrea Couto- Email: abcouto@yahoo.com.br 


\section{Introdução}

O eletrodo de diamante dopado com boro (DDB) tem se apresentado como um excelente material para ser aplicado na eletroquímica. O DDB começou a ser estudado para aplicação na eletroquímica por volta de 1980 , por apresentar propriedades que o destaca dos demais materiais utilizados convencionalmente como eletrodo. Propriedades tais como: ampla janela de potencial, baixa corrente de fundo e estabilidade física e química ${ }^{(1)}$. Devido a essas propriedades os filmes DDB têm sido estudados e aplicados como eletrodo na eletroquímica para o tratamento de água contaminada, como sensor em análises eletroquímicas, eletrólise de compostos orgânicos e inorgânicos ${ }^{(2-4)}$. Os filmes de DDB podem ter as suas propriedades aperfeiçoadas através da modificação superficial com deposição de partículas metálicas, pois é um processo que leva ao aumento da rugosidade superficial, proporcionando, desta forma, um aumento da área condutiva do eletrodo e, consequentemente, um aumento da sensibilidade. Dessa maneira, proporciona uma melhor atividade catalítica, diminuindo o sobrepotencial de algumas reações de óxido-redução ${ }^{(5,6)}$. O depósito de partículas metálicas sobre a superfície do substrato caracteriza-se por duas etapas fundamentais: a etapa de nucleação, na qual ocorre a formação dos núcleos; e a etapa de coalescência, que é caracterizada pela junção dos núcleos originando os aglomerados. Em sistemas eletroquímicos onde são utilizados como substrato um material semicondutor (como, por exemplo, o DDB) e um metal como material a ser depositado, a etapa de nucleação é um fator fundamental para esse processo de deposição. Diferentes métodos podem ser aplicados para realizar a eletrodeposição, como: potenciostático, galvanostático, eletrodeposição espontânea conhecida como electroless e a deposição pulsada. Independentemente de qual metodologia escolhida, a principal vantagem da introdução de partículas metálicas pelo processo de eletrodeposição é que a quantidade do material a ser depositado pode ser controlada com boa precisão pelo ajuste de alguns parâmetros, tais como, o potencial de deposição, a densidade de corrente aplicada, o tempo de deposição e a composição do eletrólito. Além disso, se obtém morfologias, microestruturas e composições que podem ser modificadas facilmente. Por outro lado, vários estudos confirmaram a alta eficiência do método de eletrodeposição fotoassistido ${ }^{(7,8)}$. A fotodeposição está associada ao foto efeito, o qual é observado em materiais semicondutores, assim como o do DDB. Neste contexto, aspectos ainda inexplorados sobre a deposição de partículas de cobre sobre filmes de diamante dopado com boro serão avaliados utilizando-se as técnicas de eletrodeposição assistida por iluminação ultravioleta. Será investigada a influência do nível de dopagem dos filmes na eletrodeposição fotoassistida de cobre bem como a influência do meio eletrolítico utilizando soluções com diferentes pHs (ácido e neutro).

\section{Materiais e Métodos}

Os filmes de DDB foram produzidos pela técnica CVD (Chemical Vapor Deposition) utilizando-se um reator de filamento quente sobre substrato de Si tipo p (llll 0 0), onde foram mantidos os seguintes parâmetros de crescimento: 5 filamentos de tungstênio de $125 \mu \mathrm{m}$ de diâmetro, distância entre os filamentos e o substrato $3 \mathrm{~mm}$, temperatura do substrato em $780^{\circ} \mathrm{C}$, pressão de 50 Torr, o tempo de deposição de $16 \mathrm{~h}$ e uma mistura gasosa composta por $1,5 / 98,5 \%$ de $\mathrm{CH}_{4} / \mathrm{H}_{2}$ em um fluxo total dos gases de $200 \mathrm{sccm}$ (centímetro cúbico por minuto- "standard centimeter cubic minute”). A dopagem foi realizada através de uma linha adicional de $\mathrm{H}_{2}$, que passa através do borbulhador que contém óxido de boro $\left(\mathrm{B}_{2} \mathrm{O}_{3}\right)$ dissolvido em metanol e arrasta o boro até o reator. Foram produzidos filmes de DDB com dopagem de 5000 e 30000 ppm na razão B/C. Os filmes produzidos foram caracterizados em relação à morfologia assim como em relação à estrutura. A análise morfológica foi realizada por microscopia eletrônica de varredura (MEV), utilizando um equipamento marca JEOL modelo JSM-5310. A caracterização estrutural foi realizada através da técnica de espectroscopia de espalhamento Raman; por um microscópio Renishaw 2000 com a utilização de um laser de argônio (514,5 nm). As curvas de Mott Schottky Plot (MSP) foram realizadas em $0,5 \mathrm{~mol} \mathrm{~L}^{-1} \mathrm{H}_{2} \mathrm{SO}_{4}$ no potencial entre $-0,8$ e $0,6 \mathrm{~V} \mathrm{x}$ $\mathrm{Ag} / \mathrm{AgCl} / \mathrm{KCl}_{\text {(sat) }}$, na frequência de 1,10 e $50 \mathrm{kHz}$ para três diferentes pertubações pico a pico de 10, 20 e $40 \mathrm{mV}$. Esses intervalos de variação tanto para a frequência quanto para o potencial de pertubação são importantes para avaliar a reprodutibilidade das medidas de capacitância, utilizadas para calcular concentração de portadores nos filmes de DDB. O depósito de partículas de cobre sobre os filmes de DDB foi realizado com o emprego da cronoamperometria; em um sistema Autolab PGSTAT 302 e uma 
lâmpada de Deutério modelo 60093 de $30 \mathrm{~W}$, para emissão de luz UV. Os filmes de DDB foram utilizados como eletrodo de trabalho, platina como contra eletrodo e $\mathrm{Ag} /$ $\mathrm{AgCl} / \mathrm{KCl}_{\text {(sat) }}$ como eletrodo de referência. Os depósitos de partículas de cobre foram realizados em meio neutro em $1 \mathrm{mmol} \mathrm{L}^{-1} \mathrm{CuSO}_{4}+50 \mathrm{mmol} \mathrm{L}^{-1} \mathrm{NaClO}_{4}$ e em meio ácido em uma solução composta por $1 \mathrm{mmol} \mathrm{L}^{-1} \mathrm{CuSO}_{4}$ $+50 \mathrm{mmol} \mathrm{L}^{-1} \mathrm{HClO}_{4}$. O tempo de deposição e o potencial de deposição foram fixados em 10 minutos e em - $0,6 \mathrm{~V}$, respectivamente.

\section{Resultados e Discussões}

As morfologias superficiais dos filmes de DDB com suas respectivas imagens de seção de corte (detalhe) são apresentadas na Fig.1 (a) e (b) para as dopagens de 5000 e $30000 \mathrm{ppm} \mathrm{B/C}$, respectivamente. As micrografias dos filmes com diferentes dopagens mostraram que há um recobrimento homogêneo de toda a superfície do substrato sem apresentar fissuras ou pontos de delaminação, o que caracteriza um filme de boa qualidade. Em relação à morfologia dos filmes de DDB, verifica-se que os mesmos são formados por grãos microcristalinos facetados, com orientação cristalográfica predominante (111).

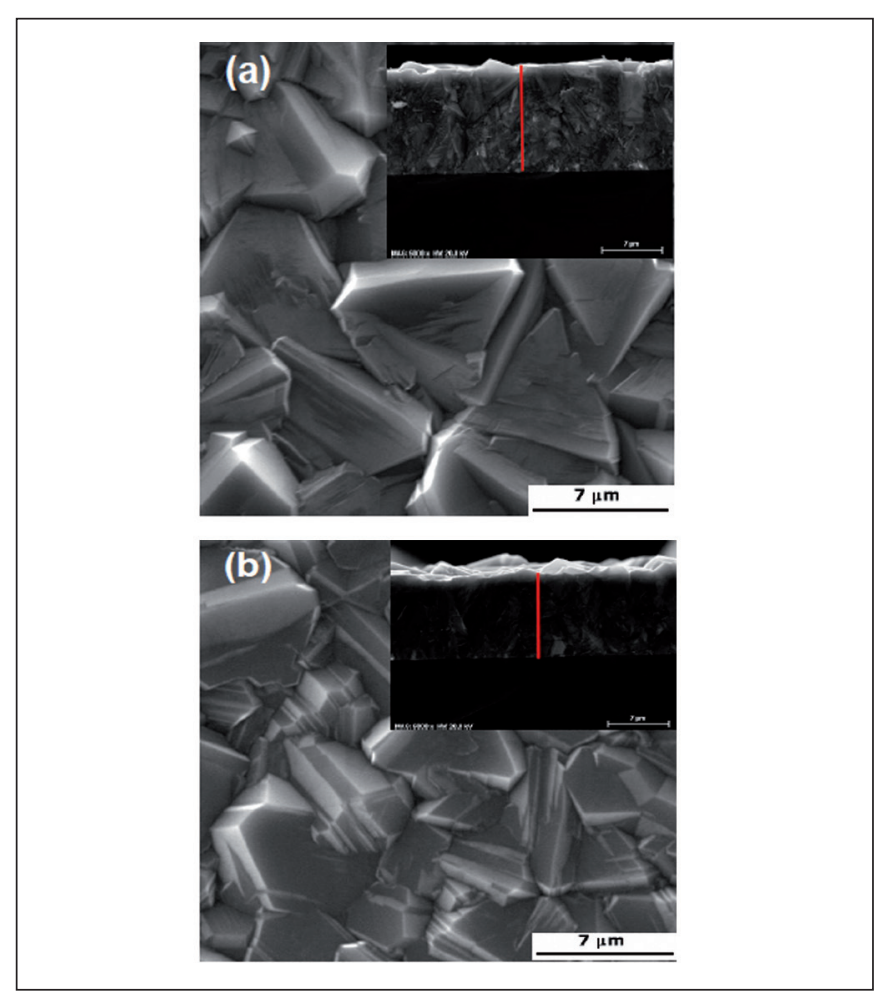

Figura 1. Imagens $\mathrm{MEV}$ da morfologia e de seção de corte dos filmes de DDB; (a) 5000 ppm B/C; (b) 30000 ppm B/C.
Observa-se que, independentemente da dopagem, os filmes apresentam tamanho de grão similar, principalmente atribuído ao longo tempo de crescimento. Este comportamento também está evidente nas imagens de seção de corte de ambos os filmes, que apresentam crescimento colunar, conforme esperado para diamante microscristalino, com valores de espessuras muito próximos, em torno de 11,6 e $11 \mu \mathrm{m}$ para os filmes (a) e (b), respectivamente. Esses resultados são coerentes considerando que a inclusão de boro na rede cristalina do diamante pode causar uma pequena variação na taxa de crescimento levando a uma grande população de pequenos grãos entre os maiores. Este efeito está relacionado ao alto nível de dopagem na amostra, proporcionando também a maior inclusão de impureza do tipo $\mathrm{sp}^{2}$, mesmo para filmes bem espessos ${ }^{(9)}$.

Para avaliar tanto a qualidade dos filmes como a influência da inclusão do dopante boro a técnica de microscopia de espalhamento Raman é bastante adequada. Os espectros dos dois tipos de DDB estudados estão apresentados na Fig. 2. Para ambos os espectros pode-se observar um pico bem definido em $1332 \mathrm{~cm}^{-1}$ que caracteriza a estrutura do diamante e as duas bandas localizadas em $500 \mathrm{~cm}^{-1} \mathrm{e}$ $1230 \mathrm{~cm}^{-1}$ que estão associadas à incorporação de boro nos filmes de diamante. Verifica-se que essas bandas tornam-se mais intensas para o filme de DDB com 30000 ppm de boro em comparação ao filme de DDB dopado com 5000 ppm de boro, devido a maior incorporação de boro na rede cristalina do diamante ${ }^{(10,11)}$. A presença da banda

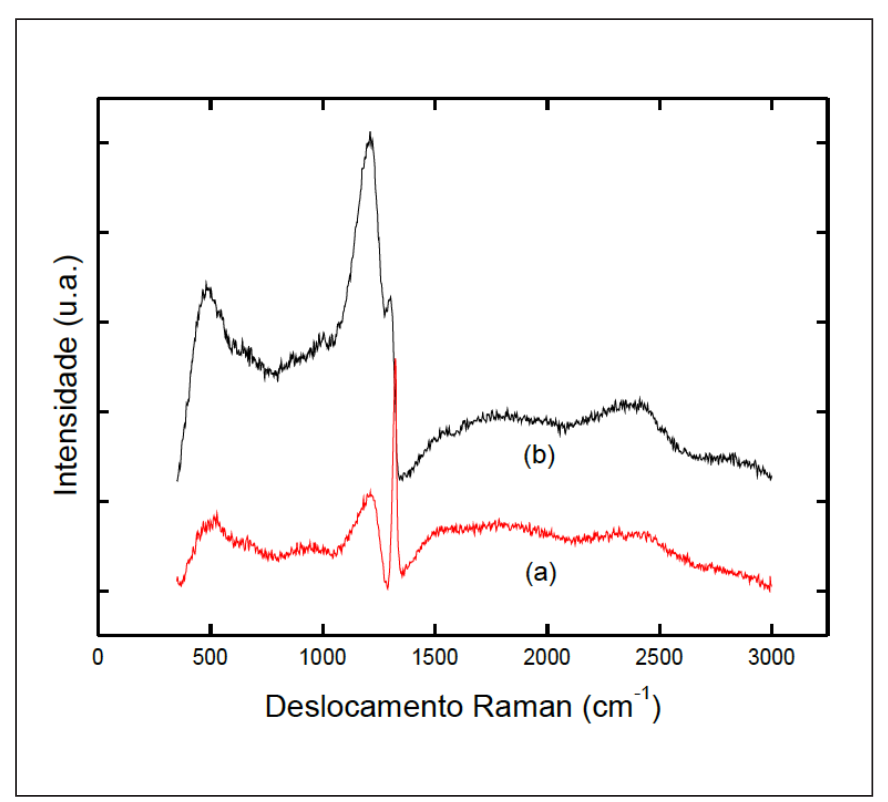

Figura 2. Espectros Raman dos filmes de DDB com diferentes razões $\mathrm{B} / \mathrm{C}$ em metanol; (a) 5000 ppm e (b) $30000 \mathrm{ppm}$. 
em $500 \mathrm{~cm}^{-1}$ é atribuída ao aumento da concentração de pares de boro que apresenta modos de vibração local ${ }^{(12)}$, enquanto que, a banda centrada em $1230 \mathrm{~cm}^{-1}$ é atribuída à interferência de Fano entre o estado discreto do fônon e a continuidade eletrônica ${ }^{(10)}$. Seguindo-se esta discussão, o pico de banda em $1230 \mathrm{~cm}^{-1}$ deve representar a combinação de picos de fônons ópticos causados pela incorporação do boro no diamante. Corroborando com os resultados de May et al. ${ }^{(12)}$, Niu et al. ${ }^{(13)}$ discutiram as vibrações eletrônicas associadas às propriedades de filmes de diamante altamente dopados e concluíram que as bandas em 500 e $1230 \mathrm{~cm}^{-1}$ podem ser ambas originárias não somente das vibrações em ligações $\mathrm{C}-\mathrm{C}$ com defeitos induzidos, mas também por vibrações do tipo B-B e B-C, respectivamente. A presença de uma banda em torno de $1580 \mathrm{~cm}^{-1}$ é atribuída ao carbono com ligações do tipo $\mathrm{sp}^{2}$, ou seja, do tipo grafite, que não está evidenciada para ambos os filmes, mostrando a boa qualidade dos mesmos. Observando-se a região de segunda ordem do espectro Raman verifica-se uma banda por volta de $2500 \mathrm{~cm}^{-1}$, conhecido como segunda ordem de vibração que corresponde a aproximadamente ao dobro da posição da banda posicionada em $1230 \mathrm{~cm}^{-1(12,12)}$.

O gráfico de Mott-Schottky consiste em um método prático para a caracterização de interfaces envolvendo semicondutores porque as medidas do potencial de banda plana (EFB) podem ser realizadas na ausência de luz ${ }^{(15)}$. De acordo com a equação de Mott-Schottky, a capacitância $\left(\mathrm{C}^{-2}\right)$ deve variar linearmente com o potencial aplicado na interface. Nesta relação, admite-se que toda a carga presente na camada de depleção é proveniente dos doadores ionizados. Portanto, um gráfico de $\mathrm{C}^{-2} \mathrm{em}$ função de $\mathrm{E}$ informa sobre o nível de dopagem no semicondutor, permitindo estimar a densidade efetiva dos portadores de carga (NA) e também o potencial de bandas planas $(\mathrm{EFB})^{(16)}$.

$$
N_{A}=\frac{2}{e \varepsilon_{0} \varepsilon}\left[d\left(C^{-2}\right) / d V\right]^{-1}
$$

onde $N_{\mathrm{A}}$ é a densidade efetiva dos portadores de carga, e é a carga do elétron; $\varepsilon_{0}$ e $\varepsilon$ representam a permissividade do vácuo e do semicondutor, respectivamente.

A Fig. 3 apresenta as curvas Mott-Schottky para os eletrodos de DDB nas duas dopagens estudadas utilizando-se $0,5 \mathrm{~mol} \mathrm{~L}^{-1} \mathrm{H}_{2} \mathrm{SO}_{4}$ e frequência de $10 \mathrm{kHz}$ e 40 $\mathrm{mV}$ de amplitude. As concentrações de portadores foram calculadas e estão entre $2,7 \times 10^{19}$ a $5,7 \times 10^{19}$ e $1,6 \times 10^{20}$ a $3,0 \times 10^{20}$, para os filmes de DDB com concentração de boro 5000 e 30000 ppm, respectivamente. Deve-se ressaltar que as medidas MSP foram realizadas em três diferentes frequências e em três diferentes amplitudes, os resultados apresentaram boa reprodutibilidade, com pouca dependência com a frequência e amplitude.

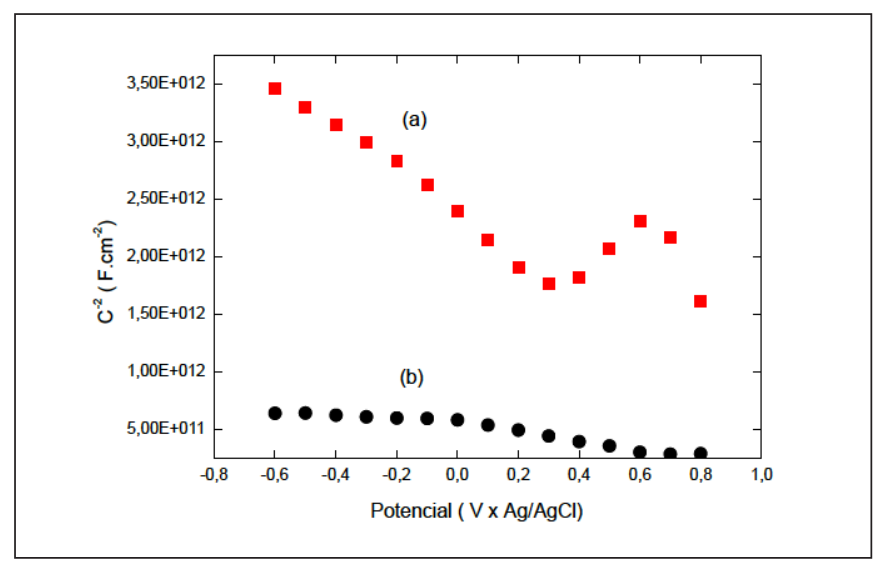

Figura 3. Curvas Mott-Schottky para os eletrodos de DDB em 0,5 mol L-1 $\mathrm{H}_{2} \mathrm{SO}_{4}$ e frequência de $10 \mathrm{kHz}$ e $40 \mathrm{mV}$ de amplitude nas duas dopagens estudadas:

(a) 5000 ppm e (b) $30000 \mathrm{ppm}$.

A fotoeletrodeposição de cobre pode ser influenciada pela dopagem do filme de DDB. Para avaliar tal influência realizou-se a fotoeletrodeposição de cobre em dois diferentes meios: neutro $\left(1 \mathrm{mmol} \mathrm{L}^{-1} \mathrm{CuSO}_{4}+50 \mathrm{mmol} \mathrm{L}_{-1} \mathrm{NaClO}_{4}\right) \mathrm{e}$ ácido $\left(1 \mathrm{mmol} \mathrm{L}^{-1} \mathrm{CuSO}_{4}+50 \mathrm{mmol} \mathrm{L}^{-1} \mathrm{HClO}_{4}\right)$ sobre filmes de DDB com concentração de boro de 5000 ppm e 30000 ppm. A análise morfológica das partículas de cobre fotoeletrodepositadas sobre filmes de DDB com diferentes dopagens foi caracterizada por MEV e está apresentada na Fig. 4.

As imagens MEV mostraram que a fotoeletodeposição das partículas de cobre sobre os filmes de DDB apresentaram de forma geral uma distribuição uniforme das partículas nas faces dos filmes e um formato arredondado. A uniformidade dos depósitos de cobre pode estar associada aos elétrons fotogerados, o qual potencializa o processo de redução do cobre sobre a superficie do DDB. Entretanto em meio ácido (Figs. 4c e 4d) a distribuição das partículas de cobre é menos eficiente quando comparados com a distribuição das partículas em meio neutro (Figs. 4a e 4b). Este comportamento pode ser atribuído a uma possível competição entre o $\mathrm{H}^{+}$e o $\mathrm{Cu}^{2+}$ pelos elétrons fotogerados, uma vez que $\mathrm{o} \mathrm{H}^{+}$também pode ser fotoeletricamente reduzido na superfície do diamante semicondutor ${ }^{(17-19)}$. Em meio neutro há uma menor quantidade de $\mathrm{H}^{+}$disponível para ocorrer a redução do mesmo gerando gás hidrogênio, portanto, os elétrons fotogerados são capturados pelos íons $\mathrm{Cu}^{2+}$, aumentando o depósito de cobre sobre a superfície do DDB. Observa-se ainda, que em meio neutro as partículas de cobre 

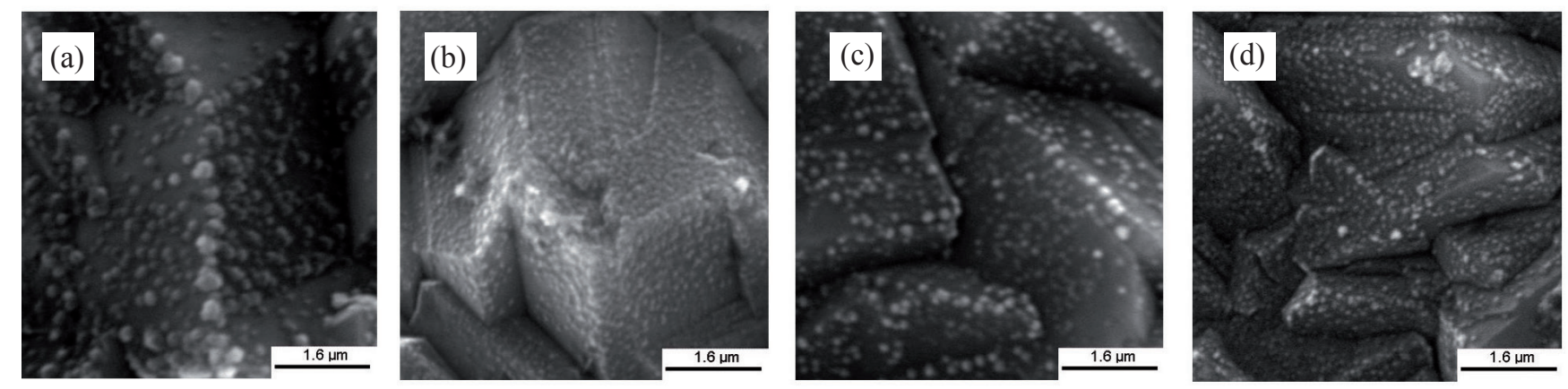

Figura 4. Imagens $\mathrm{MEV}$ do depósito de cobre sobre filmes de DDB com diferentes dopagens:

(a) $\mathrm{B} / \mathrm{C}=5000$ ppm meio neutro $\left(\mathrm{NaClO}_{4}\right)$; (b) $\mathrm{B} / \mathrm{C}=30000 \mathrm{ppm}$ meio neutro $\left(\mathrm{NaClO}_{4}\right)$; (c) $\mathrm{B} / \mathrm{C}=5000$ ppm meio ácido $\left(\mathrm{HClO}_{4}\right) ;(\mathrm{d}) \mathrm{B} / \mathrm{C}=30000 \mathrm{ppm}$ meio ácido $\left(\mathrm{HClO}_{4}\right)$.

fotoeletrodepositadas sobre o filme de DDB com concentração de boro de 30000 ppm estão distribuídas de maneira mais homogênea; quando comparadas as partículas de cobre fotoeletrodepositadas sobre o filme de DDB com concentração de boro de 5000 ppm. Essa diferença na homogeneidade pode estar relacionada com o maior número de portadores presentes nos filmes de DDB com maior dopagem. O maior número de portadores nos filmes com 30000 ppm de boro pode facilitar o processo de redução fotoeletroquímica do cobre.

$\mathrm{O}$ comportamento eletroquímico do $\mathrm{Cu}$ nos filmes com diferentes dopagens foi avaliado tanto para o meio eletrolítico neutro como para o meio eletrolítico ácido. A voltametria cíclica foi realizada utilizando-se velocidade de varredura de $100 \mathrm{mV} \mathrm{s}^{-1}$. A Fig. 5 apresenta os voltamogramas cíclicos do cobre em filmes de DDB com diferentes dopagens nos dois meios eletrolíticos estudados.

Como pode ser visualizado na Fig. 5, ao analisar o voltamograma realizado com a utilização do filme de DDB com dopagem de 5000 ppm, verifica-se que o pico catódico inicia em um potencial mais negativo em relação ao filme mais dopado em ambos os meios. Tal diferença entre os início da corrente catódica pode estar associado ao número de portadores presentes em cada filme. Portanto, o início antecipado do pico catódico no filme com dopagem de 30000 ppm pode estar relacionado ao maior número de portadores disponíveis para o processo de redução eletroquímica do $\mathrm{Cu}$, indicando uma condição energética mais favorável para o processo. Cabe ressaltar que para ambos os filmes, observa-se que o meio ácido é energeticamente menos favorável para a redução dos íons $\mathrm{Cu}$, pois a corrente catódica tem seu início retardado quando comparado com os filmes em meio neutro. Este retardo pode estar diretamente relacionado à distribuição

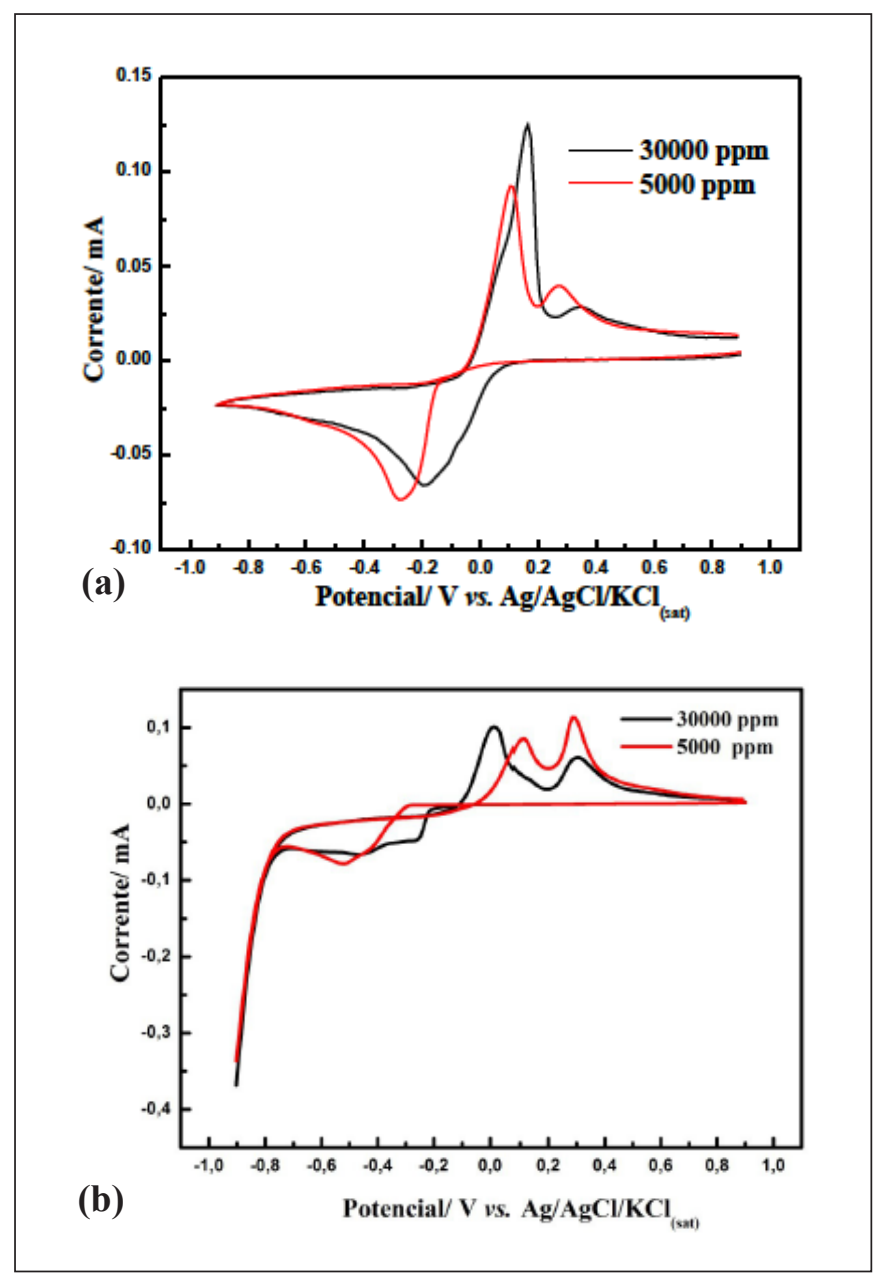

Figura 5. Voltamogramas cíclicos do cobre em filmes de DDB com diferentes dopagens; (a) meio neutro $\left(\mathrm{NaClO}_{4}\right)$ e (b) meio ácido $\left(\mathrm{HClO}_{4}\right)$.

menos eficiente das partículas de cobre sobre o DDB em meio ácido, como observado nas imagens MEV descritas acima. Portanto, o melhor processo de fotoeletrodeposição 
de cobre ocorre com a utilização de uma solução neutra e um filme de DDB de 30000 ppm. Este resultado pode ser atribuído a própria condição energética que é mais favorável a redução do cobre, como pode ser observado nos voltamogramas cíclicos (Fig. 5). Além disto, em meio neutro há uma menor competição pelos elétrons fotogerados entre o $\mathrm{H}^{+}$e o $\mathrm{Cu}^{2+}$, uma vez que neste meio há uma menor quantidade de $\mathrm{H}^{+}$disponível para ocorrer a redução do mesmo gerando gás hidrogênio. Portanto, os elétrons fotogerados são capturados pelos íons $\mathrm{Cu}^{2+}$, aumentando o depósito de cobre sobre a superfície do DDB.

\section{Conclusões}

Os filmes de DDB produzidos apresentaram uma boa qualidade. A microscopia eletrônica de varredura revelou que os filmes cresceram sobre toda a superfície do substrato sem a presença de fissuras e com uma orientação cristalográfica predominante (111). A espectroscopia Raman apresentou o pico característico do diamante em $1332 \mathrm{~cm}^{-1}$, bem como as bandas referentes à

\section{Referências}

1. Banda, G.R.S.; Einaga, Y.; Martines-Huitle,C.A.; International Journal of Electrochemistry 2012 (2011) 1.

2. Panizza, M.; Cerisola, G.; Electrochemica Acta 51 (2005) 191.

3. Raghu, S.; Lee, C.W.; Chellammal, S.; Palanichamy, S.; Basha, C.A.; Journal of Hazardous Materials 171 (2009) 748.

4. Lacasa, E.; Llanos, J.; Cañizares, P.; Rodrigo, M.A.; Chemical Engineering Journal 184 (2012) 66.

5. Welch, C.M.; Hyde, M.E.; Banks, C.E.; Compton, R.G., Analytical Science 21 (2005) 1421.

6. Couto, A.B.; Santos, L.C.D.; Matsushima, J.T.; Baldan, M.R.; Ferreira, N.G.; Applied Surface Science 257 (2011) 10141.

7. Yoshihara, S.; Shinozaki, K.; Shirakashi, T.; Hashimoto, K.; Tryk, D. A.; Fujishima, A. ; Electrochimica Acta 44 (1999) 2711.

8. Wu, T.; Li, Y.; Chu, M.; Handbook of photochemistry and photobiology, American Scientific Publishers, 2003.

9. Ferreira, N.G.; Silva, L.L.G.; Corat, E.J.; Trava-Airoldi, V.J.; Diamond and Related Materials 11 (2002) 1523.

10. Zhang, R.J.; Lee, S.T.; Lam, Y.W.; Diamond and Related Materials 5 (1996) 1288.

11. Migliorini, F.L.; Produção e caracterização de eletrodos de diamante dopados com boro crescidos sobre titânio, incorporação de átomos de boro na rede cristalina do diamante $\left(500 \mathrm{~cm}^{-1}\right.$ e $\left.1200 \mathrm{~cm}^{-1}\right)$. Das curvas de Mott-Schottky Plot foi possível estimar a concentração de portadores para os filmes de DDB. Os resultados mostraram que a deposição das partículas de cobre sobre os filmes de DDB através da fotoeletrodeposição em meio neutro é melhor quando comparada à fotoeletrodeposição em meio ácido. A influência da dopagem na eletrodeposição revelou que as partículas de cobre fotoeletrodepositadas sobre o filme de DDB com concentração de boro 30000 ppm estão distribuídas de maneira mais homogênea; quando comparadas às partículas de cobre fotoeletrodepositadas sobre o filme de DDB com concentração de boro de 5000 ppm. Essa diferença na homogeneidade dos depósitos pode estar relacionada com o maior número de portadores presentes nos filmes de DDB com maior dopagem.

\section{Agradecimentos}

À FAPESP (Processo 2010/05391-0), ao CNPQ e CAPES pelo apoio financeiro.

aplicados na degradação de corante têxtil. Dissertação de Mestrado (2011). Instituto Nacional de Pesquisas Espaciais- INPE, São José dos Campos (SP).

12. May, P.W.; Ludlow, W.J.; Hannaway, M.; Heard, P.J.; Smith, J.A.; Rossner, K.N.; Diamond and Related Materials 17 (2008) 105

13. Niu, L.; Zhu, J.Q.; Han, X.; Tan, M., Gao, W.; Du, S.Y.; Phys. Lett. A 373 (2009) 2494.

14. Bernard, M.; Baron, C.; Deneuville, A.; Diamond and Related Materials13 (2004) 896.

15. Silva, L. L. G.; Eletrodos em diamante CVD para estudos eletroquímicos. Tese de Doutorado (2001). Instituto Tecnológico de Aeronáutica, São José dos Campos (SP).

16. Ferreira, N.G.; Silva, L.L.G.; Corat, E.J.; Trava-Airoldi, V.J.; Iha, K.; Brazilian Journal of Physics 29 (1999) 760.

17. Boonma, L.; Yano, T.; Tryk, D.A.; Hashimoto, K.; Fujishima,A.; J. Electrochem. Soc.144 (1997) L142.

18. Rao, T.N.; Tryk, D.A.; Hashimoto, K.; Fujishima,A.; J. Electrochem. Soc. 146 (1999) 680.

19. Couto, A.B.; Ribeiro, M.C.E.; Migliorini, F.L.; Ferreira, N.G.;Baldan, M.R., Diamond and Related Materials 38 (2013) 104 